TITLE:

\title{
Exact Identification of the Structure of a Probabilistic Boolean Network from Samples
}

\section{AUTHOR(S):}

Cheng, Xiaoqing; Mori, Tomoya; Qiu, Yushan; Ching, Wai-Ki; Akutsu, Tatsuya

\section{CITATION:}

Cheng, Xiaoqing ... [et al]. Exact Identification of the Structure of a Probabilistic Boolean Network from Samples. IEEE/ACM Transactions on Computational Biology and Bioinformatics 2016, 13(6): 1107-1116

ISSUE DATE:

2016-11-01

URL:

http://hdl.handle.net/2433/252326

\section{RIGHT:}

(c) 2016 IEEE. Personal use of this material is permitted. Permission from IEEE must be obtained for all other uses, in any current or future media, including reprinting/republishing this material for advertising or promotional purposes, creating new collective works, for resale or redistribution to servers or lists, or reuse of any copyrighted component of this work in other works.; この論文は出版社版でありません。引用の際には出版社版をご確認ご利用ください。; This is not the published version. Please cite only the published version. 


\title{
Exact Identification of the Structure of a Probabilistic Boolean Network from Samples
}

\author{
Xiaoqing Cheng, Tomoya Mori, Yushan Qiu, Wai-Ki Ching, and Tatsuya Akutsu, Member, IEEE
}

\begin{abstract}
We study the number of samples required to uniquely determine the structure of a probabilistic Boolean network (PBN), where PBNs are probabilistic extensions of Boolean networks. We show via theoretical analysis and computational analysis that the structure of a PBN can be exactly identified with high probability from a relatively small number of samples for reasonably wide classes of PBNs of bounded indegree. On the other hand, we also show that there exist classes of PBNs for which it is impossible to uniquely determine the structure of a PBN from samples.
\end{abstract}

Index Terms—probabilistic Boolean networks, genetic networks, network inference, sample complexity

\section{INTRODUCTION}

$\mathrm{V}$ ARIOUS kinds of mathematical models have been utilized for understanding dynamical behavior of biological systems. Among them, the Boolean network (BN) is a simple but well-studied model, which was proposed by Kauffman in 1969 as a model of gene regulatory networks [1], [2]. In a BN, each node takes a Boolean value, 0 or 1 , at each time step, and the states of all nodes are updated synchronously according to Boolean functions assigned to nodes, where each node corresponds to a gene, and 1 and 0 mean that genes are active and inactive, respectively. In spite of the simplicity of the model, BNs exhibit complex behavior and thus extensive studies have been done in order to understand their behavior. For example, many studies have been done on the distribution of attractors [3], [4], [5] and the robustness against perturbations [6], [7].

In order to study realistic BNs, we need to infer the structures of BNs from real data such as gene expression time series data. Therefore, extensive studies have been done on inference of BNs from gene expression data [8], [9], [10], [11]. It is known that a BN with $n$ nodes is uniquely determined with high probability from randomly selected $O(\log n)$ state-transition samples (i.e., $O(\log n)$ random arcs in the state transition diagram) if the maximum indegree (i.e., the maximum number of input nodes) is bounded by a constant [9], where $\log n$ stands for $\log _{2} n$ in this paper. This is an interesting result because the possible number of BNs with $n$ nodes is $2^{n 2^{n}}$, and $2^{n}$ samples are needed to uniquely specify a $\mathrm{BN}$ if there is no constraint on the structure of a $\mathrm{BN}$ (i.e., there is one-to-one correspondence between BNs and state transition diagrams).

Although BNs are a deterministic model, biological systems contain intrinsic stochasticity and observed data include noise. Therefore, BNs have been extended for in-

- X. Cheng, Y. Qiu, and W-K. Ching are with Department of Mathematics, The University of Hong Kong, Pokfulam Road, Hong Kong, China. E-mail: mechxqxiaofen@gmail.com, $\{u 3001131, w c h i n g\} @ h k u . h k$,

- T. Mori and T. Akutsu are with Bioinformatics Center, Institute for Chemical Research, Kyoto University, Gokasho, Uji, Kyoto 611-0011, Japan. E-mail: \{tmori,takutsu\}@kuicr.kyoto-u.ac.jp.

X.C. and T.M. contributed equally to this work.

Manuscript received XXX XX, XXXX; revised XXX XX, XXXX. cluding noise [13], [14], [15]. Among them, the probabilistic Boolean network (PBN) model has attracted much attention because of its simplicity, flexibility, and relations to Markov chains and Bayesian networks [16], [17]. In a PBN, multiple Boolean functions can be assigned to each node and one function is randomly selected at each time step according to the prescribed probability distribution. Extensive studies have been done on control and simulation of PBNs [18], [19], [20], [21], [22]. Several studies have also been done on inference of PBNs [16], [22], [23].

However, to our knowledge, there is no result on the sample complexity analogous to one for BNs. Although there exist some studies on related models (e.g., Bayesian networks) [14], [24], [25], results in [24], [25] are not on exact identification but on approximate identification, and the model in [14] is far from PBNs. Therefore, in this paper, we study the number of samples required to exactly identify the structure (i.e., a set of Boolean functions assigned to each node) of a PBN. We show that there are cases for which it is impossible to uniquely determine a PBN from samples. This result is reasonable because PBNs are stochastic systems. Interestingly, we also show that the structure of a PBN can be identified with high probability from $O(\log n)$ samples for reasonably wide classes of PBNs of bounded indegree.

\section{Probabilistic Boolean Network}

A $\mathrm{BN}$ is a directed network with $n$ nodes $x_{1}, \ldots, x_{n}$. Each node takes either 0 or 1 at each time step, and the state of $x_{i}$ at time step $t$ is denoted by $x_{i}(t)$. The states of all nodes are updated simultaneously according to Boolean functions assigned to nodes. Let $f^{(i)}$ denote the Boolean function assigned to node $x_{i}, I N\left(f^{(i)}\right)$ denote a set of input nodes for $f^{(i)}$, and $\hat{f}^{(i)}$ denote its extension to all nodes $x_{1}, \ldots, x_{n}$. Let $\mathbf{x}(t)$ denote the global state of a BN at time $t$, (i.e., $\mathbf{x}(t)=\left(x_{1}(t), \ldots, x_{n}(t)\right)$. Then, the dynamics of the $\mathrm{BN}$ is given by $\mathbf{x}(t+1)=\left(\hat{f}^{(1)}(\mathbf{x}(t)), \ldots, \hat{f}^{(n)}(\mathbf{x}(t))\right)$. For example, suppose $I N\left(f^{(i)}\right)=\left\{x_{j}, x_{k}\right\}$. Then, $x_{i}(t+1)$ is determined by $x_{i}(t+1)=f^{(i)}\left(x_{j}(t), x_{k}(t)\right)$, which can also be written as $x_{i}(t+1)=\hat{f}^{(i)}(\mathbf{x}(t))$. 


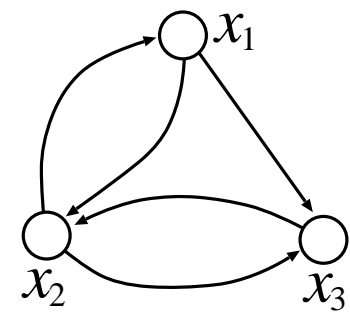

\begin{tabular}{|l|l|c|}
\hline & \multicolumn{1}{|c|}{ Boolean Function } & Prob. \\
\hline$f_{1}^{(1)}$ & $x_{1}(t+1)=x_{2}(t)$ & 0.8 \\
$f_{2}^{(1)}$ & $x_{1}(t+1)=\overline{x_{2}(t)}$ & 0.2 \\
\hline$f_{1}^{(2)}$ & $x_{2}(t+1)=x_{1}(t) \wedge \overline{x_{3}(t)}$ & 0.7 \\
$f_{2}^{(2)}$ & $x_{2}(t+1)=x_{3}(t)$ & 0.3 \\
\hline$f_{1}^{(3)}$ & $x_{3}(t+1)=x_{1}(t) \wedge \overline{x_{2}(t)}$ & 1.0 \\
\hline
\end{tabular}

Fig. 1. Example of PBN.

A PBN is an extension of a BN. It is also a directed network with $n$ nodes. As mentioned before, in a PBN, multiple Boolean functions can be assigned per node and one function is randomly selected at each time step according to the prescribed probability distribution. Let $\left\{f_{1}^{(i)}, \ldots, f_{m_{i}}^{(i)}\right\}$ be a set of Boolean functions assigned to node $x_{i}$, and $c_{j}^{(i)}$ denote the selection probability of $f_{j}^{(i)}$, where $\sum_{j=1}^{m_{i}} c_{j}^{(i)}=1$ must be satisfied. It is to be noted that $I N\left(f_{j}^{(i)}\right) \neq I N\left(f_{k}^{(i)}\right)$ is allowed. Then, $x_{i}(t+1)$ is determined by

$$
x_{i}(t+1)=\hat{f}_{j}^{(i)}(\mathbf{x}(t)) \text { with probability } c_{j}^{(i)},
$$

where selection of $f_{j}^{(i)}$ is independent of selections in previous time steps and selections for other nodes. Since selections of Boolean functions are done for all nodes simultaneously and independently, dynamics of the whole PBN is represented by the transition probabilities from global states at time $t$ to those at time $t+1$.

An example of PBN is given in Fig. 1. Suppose that $\mathbf{x}(t)=(0,0,0)$. If $\left(f_{1}^{(1)}, f_{1}^{(2)}, f_{1}^{(3)}\right)$ is selected with probability $0.8 \times 0.7=0.56, \mathbf{x}(t+1)=(0,0,0)$ holds. Similarly, if $\left(f_{1}^{(1)}, f_{2}^{(2)}, f_{1}^{(3)}\right)$ is selected with probability $0.8 \times 0.3=0.24, \mathbf{x}(t+1)=(0,0,0)$ holds. On the other hand, if $\left(f_{2}^{(1)}, f_{1}^{(2)}, f_{1}^{(3)}\right)$ is selected with probability $0.2 \times 0.7=0.14$ or $\left(f_{2}^{(1)}, f_{2}^{(2)}, f_{1}^{(3)}\right)$ is selected with probability $0.2 \times 0.3=0.06, \mathbf{x}(t+1)=(1,0,0)$ holds. Therefore, we have the following transition probabilities:

$$
\begin{aligned}
& \operatorname{Prob}(\mathbf{x}(t+1)=(0,0,0) \mid \mathbf{x}(t)=(0,0,0))=0.8, \\
& \operatorname{Prob}(\mathbf{x}(t+1)=(1,0,0) \mid \mathbf{x}(t)=(0,0,0))=0.2,
\end{aligned}
$$

where the probabilities of the other transitions from $(0,0,0)$ is 0 .

\section{TWO MODELS}

In this paper, we focus on PBNs in which two different Boolean functions are assigned to each node. Therefore, a PBN always means such one unless otherwise stated.

For a $0-1$ bit vector $\mathbf{a}, \mathbf{a}_{+i}$ and $\mathbf{a}_{-i}$ denote the bit vector obtained from a by setting $i$-th bit of a to be 1 and 0 , respectively, and $\mathbf{a}[i]$ denotes the value of $i$-th bit (i.e., 0 1 value corresponding to $\left.x_{i}\right)$. We say that $x_{i}$ is relevant in $f$ if there exists a 0-1 assignment a such that $\hat{f}\left(\mathbf{a}_{+i}\right) \neq \hat{f}\left(\mathbf{a}_{-i}\right)$. When we consider a function $f$ on $K$ variables, we assume that all $K$-input variables are relevant in $f$, and $K$ is called the degree of $f(K=|I N(f)|)$.

We focus on one output node because pairs of Boolean functions can be identified independently for distinct output nodes. In the following, the target output node is denoted as $y$ (i.e., $y=x_{i}$ for some $i$ ). A sample is defined by an assignment of $0-1$ values to $x_{1}, \ldots, x_{n}$ and $y$, and is represented by a pair $(\mathbf{a}, b)$ of $n$-dimensional 0-1 input vector a and 0-1 (output) value $b$, where a corresponds to the global state at time $t$ and $b$ corresponds to the state of node $y$ at time $t+1$.

We say that a Boolean function $f$ and a sample $(\mathbf{a}, b)$ are consistent if $\hat{f}(\mathbf{a})=b$. We also say that a pair of Boolean functions $\left(f_{1}, f_{2}\right)$ and a sample are consistent if $\hat{f}_{1}(\mathbf{a})=b$ or $\hat{f}_{2}(\mathbf{a})=b$ holds. If a Boolean function or a pair of Boolean functions is consistent with every element in a set $S$ of samples, it is also consistent with $S$. We say that $\left(f_{1}, f_{2}\right)$ and $S$ are strongly consistent if $\left(f_{1}, f_{2}\right)$ and $S$ are consistent and all possible consistent input/output pairs on $\left(I N\left(f_{1}\right) \cup I N\left(f_{2}\right),\{y\}\right)$ appear in $S$. It is seen from the definitions that if $\left(f_{1}, f_{2}\right)$ is strongly consistent with $S$, it is also consistent with $S$.

In the following, $\left(f_{1}, f_{2}\right)$ is identified with $\left(f_{2}, f_{1}\right)$, and a set of pairs of Boolean functions is referred as a class of $P B N s$. Furthermore, for simplicity, we assume that a in each sample is selected uniformly at random, and choice of $f_{1}$ and $f_{2}$ is also done uniformly at random. However, all the combinatorial results are independent from the probability distribution.

When analyzing the sample complexity, we assume that each sample is generated according to some underlying pair $\left(f_{1}, f_{2}\right)$ of Boolean functions. Precisely, we assume that a sample is generated by a random 0-1 assignment to $n$ variables and the corresponding value of $f_{1}$ or $f_{2}$. A set of samples is obtained by independent execution of this generation process where duplicate samples are unified.

We consider two models: full information model (FIM) and partial information model (PIM). In FIM, we say that a pair of Boolean functions $\left(f_{1}, f_{2}\right)$ is identified from a set of samples $S$ if $\left(f_{1}, f_{2}\right)$ is the only one pair in the target class of Boolean functions that is strongly consistent with $S$. On the other hand, in PIM, we say that a pair of Boolean functions $\left(f_{1}, f_{2}\right)$ is identified from a set of samples $S$ if $\left(f_{1}, f_{2}\right)$ is the only one pair in the target class of Boolean functions that is consistent with $S$. In either model, we say that a class $C$ of PBNs is identifiable from samples if, for any pair $\left(f_{1}, f_{2}\right)$ in $C$, there exists a sample set $S$ such that $\left(f_{1}, f_{2}\right)$ is identified from $S$.

Proposition 1. If a class $C$ of PBNs is identifiable from samples under PIM, $C$ is also identifiable from samples under FIM.

Proof. Let $\left(f_{1}, f_{2}\right)$ be the correct pair. Since $\left(f_{1}, f_{2}\right)$ can be identified under PIM, there exists a set $S$ of samples for which only $\left(f_{1}, f_{2}\right)$ is consistent with $S$ under class $C$.

For any such $S$, there exists $S^{\prime} \supseteq S$ such that $\left(f_{1}, f_{2}\right)$ is strongly consistent with $S^{\prime}$. Then, all other pairs are inconsistent with $S^{\prime}$ because they are inconsistent with $S$.

This proposition implies that if $C$ is not identifiable under FIM, it is not identifiable under PIM. It is straightforward to see that the following propositions hold.

Proposition 2. A class $C$ of PBNs is identifiable from samples under PIM if and only if for any two different pairs $\left(f_{1}, f_{2}\right)$ and $\left(f_{3}, f_{4}\right)$ in $C$ there exists a 0-1 assignment a such that $\left|\left\{\hat{f}_{1}(\mathbf{a}), \hat{f}_{2}(\mathbf{a})\right\}\right|>\left|\left\{\hat{f}_{3}(\mathbf{a}), \hat{f}_{4}(\mathbf{a})\right\}\right|$ or $\hat{f}_{1}(\mathbf{a})=\hat{f}_{2}(\mathbf{a}) \neq$ $\hat{f}_{3}(\mathbf{a})=\hat{f}_{4}(\mathbf{a})$ holds. 
Proposition 3. A class $C$ of PBNs is identifiable from samples under FIM if and only if for any two different pairs $\left(f_{1}, f_{2}\right)$ and $\left(f_{3}, f_{4}\right)$ in $C$ there exists a 0-1 assignment a such that $\left\{\hat{f}_{1}(\mathbf{a}), \hat{f}_{2}(\mathbf{a})\right\} \neq\left\{\hat{f}_{3}(\mathbf{a}), \hat{f}_{4}(\mathbf{a})\right\}$ holds.

Here, we show classes of PBNs that are not identifiable from samples. Suppose that $C$ is a set of pairs of oneinput Boolean functions. That is, an element of $C$ has the form of either $\left(x_{i}, x_{j}\right),\left(x_{i}, \overline{x_{j}}\right),\left(\overline{x_{i}}, \overline{x_{j}}\right)$. Then, this class is not identifiable from samples under either model since $\left\{x_{i}, \overline{x_{i}}\right\}=\left\{x_{j}, \overline{x_{j}}\right\}=\{0,1\}$ holds for any $x_{i}, x_{j}$. It should be noted that if samples are generated according to $\left(x_{i}, \overline{x_{i}}\right)$, the value of $y$ is determined as if it were completely at random and thus we cannot identify input node(s). This idea can be generalized to the class of pairs of AND functions (resp., OR functions) of degree 2.

Proposition 4. The class of pairs of AND functions (resp., OR functions) of degree 2 is not identifiable from samples under PIM or FIM.

Proof. Let $n=3$. Consider two pairs of Boolean functions $\left(f_{1}, f_{2}\right)=\left(x_{1} \wedge x_{2}, x_{1} \wedge \overline{x_{2}}\right)$ and $\left(f_{3}, f_{4}\right)=\left(x_{1} \wedge x_{3}, x_{1} \wedge\right.$ $\left.\overline{x_{3}}\right)$. Then, $\left\{\hat{f}_{1}(\mathbf{a}), \hat{f}_{2}(\mathbf{a})\right\}=\left\{\hat{f}_{3}(\mathbf{a}), \hat{f}_{4}(\mathbf{a})\right\}$ holds for all $\mathbf{a} \in$ $\{0,1\}^{3}$ as shown below, where " 0,1 " means that we have both outputs.

\begin{tabular}{|c|cccccccc|}
\hline$x_{1}$ & 0 & 0 & 0 & 0 & 1 & 1 & 1 & 1 \\
\hline$x_{2}$ & 0 & 0 & 1 & 1 & 0 & 0 & 1 & 1 \\
\hline$x_{3}$ & 0 & 1 & 0 & 1 & 0 & 1 & 0 & 1 \\
\hline$f_{1}$ & 0 & 0 & 0 & 0 & 0 & 0 & 1 & 1 \\
\hline$f_{2}$ & 0 & 0 & 0 & 0 & 1 & 1 & 0 & 0 \\
\hline Output & 0 & 0 & 0 & 0 & 0,1 & 0,1 & 0,1 & 0,1 \\
\hline$f_{3}$ & 0 & 0 & 0 & 0 & 0 & 1 & 0 & 1 \\
\hline$f_{4}$ & 0 & 0 & 0 & 0 & 1 & 0 & 1 & 0 \\
\hline
\end{tabular}

Next, we show another example to explain why we consider two models. Let $C=\left\{\left(x_{1}, x_{1} \vee x_{2}\right),\left(x_{1} \wedge x_{2}, x_{1} \vee x_{2}\right)\right\}$. Consider the following two sample sets $S_{1}$ and $S_{2}$, where " 0,1 " means that we have both outputs.

\begin{tabular}{|c|cccc|}
\hline$x_{1}$ & 0 & 0 & 1 & 1 \\
\hline$x_{2}$ & 0 & 1 & 0 & 1 \\
\hline Output in $S_{1}$ & 0 & 0,1 & 1 & 1 \\
\hline Output in $S_{2}$ & 0 & 0,1 & 0,1 & 1 \\
\hline
\end{tabular}

Then, the former pair is strongly consistent with $S_{1}$, but is not consistent with $S_{2}$. The latter pair is strongly consistent with $S_{2}$ and is consistent with $S_{1}$. Therefore, the class $C$ is identifiable from samples under FIM, but is not under PIM. However, when $S_{1}$ is given, only the former pair is strongly consistent. After a sample $(10,0)$ is added (i.e., the set of sample becomes $S_{2}$ ), the former pair is no more strongly consistent but the latter pair newly becomes strongly consistent. Therefore, under FIM, we may not be able to know whether the current set of samples is enough. On the other hand, we can know under PIM whether the current set of samples is enough. However, as shown later, a much wider class of PBNs is identifiable under FIM. Therefore, it might be better to use FIM if a relatively large number of samples are available.

\section{Partial Information Model}

For PIM, we focus on the case in which each of $f_{1}$ and $f_{2}$ is an AND or OR function of fixed degree $K$. Based on Proposition 4, we assume that both $x_{i}$ and $\overline{x_{i}}$ do not appear in $f_{1}$ and $f_{2}$ (i.e., if $x_{i}$ appears in $f_{1}$, then $\overline{x_{i}}$ cannot appear in $f_{2}$ ) for any variable $x_{i}$. Such a pair is called an admissible AND/OR pair. We will show that the class of admissible AND/OR pairs of fixed degree $K(K>1)$ is identifiable from samples under PIM. From Proposition 2, it is enough to show that for any $\left(f_{3}, f_{4}\right) \neq\left(f_{1}, f_{2}\right)$, there exists a such that $\left|\left\{\hat{f}_{1}(\mathbf{a}), \hat{f}_{2}(\mathbf{a})\right\}\right|>\left|\left\{\hat{f}_{3}(\mathbf{a}), \hat{f}_{4}(\mathbf{a})\right\}\right|$ or $\hat{f}_{1}(\mathbf{a})=\hat{f}_{2}(\mathbf{a}) \neq \hat{f}_{3}(\mathbf{a})=\hat{f}_{4}(\mathbf{a})$ holds. In the following, such an assignment is called a witness for $\left\langle\left(f_{1}, f_{2}\right),\left(f_{3}, f_{4}\right)\right\rangle$. It is to be noted that a witness for $\left\langle\left(f_{1}, f_{2}\right),\left(f_{3}, f_{4}\right)\right\rangle$ is not necessarily a witness for $\left\langle\left(f_{3}, f_{4}\right),\left(f_{1}, f_{2}\right)\right\rangle$.

We consider admissible AND/OR functions of degree $K$ in Theorem 1 . In the proof, we consider several cases depending on types (AND/OR) of $f_{i}$ s. The basic idea is common as follows. If there exists an assignment $\mathbf{a}$ such that $\hat{f}_{3}(\mathbf{a})=\hat{f}_{4}(\mathbf{a})=1$ and $\hat{f}_{1}(\mathbf{a})=0 \vee \hat{f}_{2}(\mathbf{a})=0$ (or, $\hat{f}_{3}(\mathbf{a})=\hat{f}_{4}(\mathbf{a})=0$ and $\left.\hat{f}_{1}(\mathbf{a})=1 \vee \hat{f}_{2}(\mathbf{a})=1\right)$ hold, $\mathbf{a}$ is a witness for $\left\langle\left(f_{1}, f_{2}\right),\left(f_{3}, f_{4}\right)\right\rangle$. To be more precise, we consider the case that all $f_{i}$ s are AND functions, $I N\left(f_{1}\right)=$ $I N\left(f_{3}\right)$, and $I N\left(f_{2}\right)=I N\left(f_{4}\right)$. Since $\left(f_{1}, f_{2}\right) \neq\left(f_{3}, f_{4}\right)$, we can assume without loss of generality (w.l.o.g.) that there exists a variable $x_{i}$ such that $x_{i}$ appears negatively in $f_{1}$ but positively in $f_{3}$. Since $\left(f_{3}, f_{4}\right)$ is an admissible pair, there exists an assignment a such that $\hat{f}_{3}(\mathbf{a})=\hat{f}_{4}(\mathbf{a})=1$. Then, $\hat{f}_{1}(\mathbf{a})=0$ holds for any such $\mathbf{a}$.

Theorem 1. The class of PBNs of admissible AND/OR functions of degree $K$ is identifiable from samples under PIM.

Proof. We consider five cases depending on AND/OR types of $f_{i}(i=1, \ldots, 4)$, which cover all cases by taking symmetric cases (i.e., exchange of AND and OR) into account. For each case, we prove that there exists a witness for $\left\langle\left(f_{1}, f_{2}\right),\left(f_{3}, f_{4}\right)\right\rangle$.

Case 1: all $f_{i} \mathrm{~s}(i=1, \ldots, 4)$ are $\operatorname{AND}$, and $\left(f_{3}, f_{4}\right) \neq$ $\left(f_{1}, f_{2}\right)$.

It is enough to consider the following two cases (see also Fig. 2).

Case 1-A: $I N\left(f_{1}\right)=I N\left(f_{3}\right)$ and $I N\left(f_{2}\right)=I N\left(f_{4}\right)$. Since $\left(f_{1}, f_{2}\right) \neq\left(f_{3}, f_{4}\right)$, we can assume w.l.o.g. that there exists a variable $x_{i}$ such that $x_{i}$ appears negatively in $f_{1}$ but positively in $f_{3}$. Since $\left(f_{3}, f_{4}\right)$ is an admissible pair, there exists an assignment a such that $\hat{f}_{3}(\mathbf{a})=\hat{f}_{4}(\mathbf{a})=1$. Then, $\hat{f}_{1}(\mathbf{a})=0$ holds for any such $\mathbf{a}$, and thus $\mathbf{a}$ is a witness.

Case 2-A: $I N\left(f_{1}\right) \neq I N\left(f_{3}\right)$ and $I N\left(f_{1}\right) \neq I N\left(f_{4}\right)$.

We assume w.l.o.g. from the symmetry and the discussion in Case 1-A that all variables appear positively in $f_{1}, f_{2}, f_{3}$, and $f_{4}$. Let $x_{i} \in I N\left(f_{3}\right) \backslash I N\left(f_{1}\right)$ and $x_{j} \in I N\left(f_{4}\right) \backslash I N\left(f_{1}\right)$, where $x_{i}=x_{j}$ is allowed, and $X_{1} \backslash X_{2}$ denotes the set $\left\{x \mid x \in X_{1}, x \notin X_{2}\right\}$. Consider an assignment a such that 0 is assigned to $x_{i}$ and $x_{j}$, and 1 is assigned to the other variables. Then, $\hat{f}_{3}(\mathbf{a})=\hat{f}_{4}(\mathbf{a})=0$ and $\hat{f}_{1}(\mathbf{a})=1$ hold.

Case 2: $f_{1}, f_{2}, f_{3}$ are AND functions, and $f_{4}$ is an OR function. 

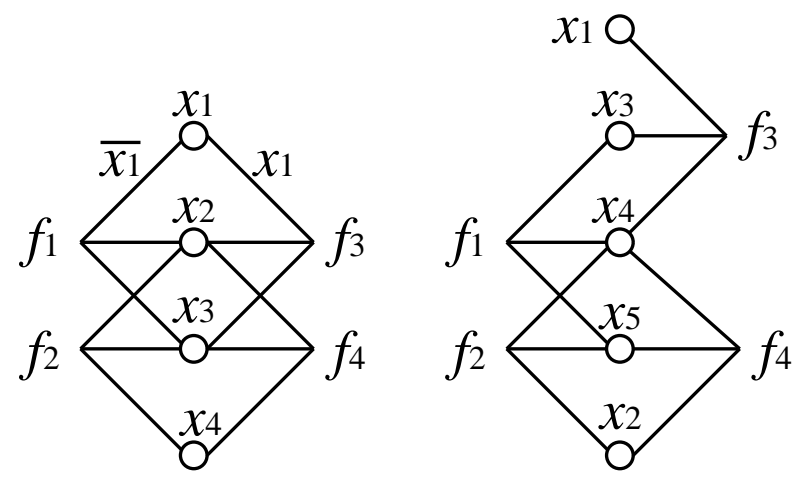

Case 1-A

Fig. 2. Illustration of two subcases of Case 1 in the proof of Theorem 1. In Case 1-A, $\hat{f}_{3}(\mathbf{a})=\hat{f}_{4}(\mathbf{a})=1$ but $\hat{f}_{1}(\mathbf{a})=0$ hold for an assignment $\mathbf{a}=(1,1,1,1)$. In Case 1-B, $f_{3}=f_{4}=0$ but $f_{1}=1$ for an assignment $\hat{f}_{3}(\mathbf{a})=\hat{f}_{4}(\mathbf{a})=0$ but $\hat{f}_{1}(\mathbf{a})=1$ hold for an assignment $\mathbf{a}=(0,0,1,1,1)$.

We assume w.l.o.g. that $I N\left(f_{2}\right) \neq I N\left(f_{3}\right)$. We further assume w.l.o.g. that all variables appear positively in $f_{1}, f_{2}$, $f_{3}$, and $f_{4}$.

Let $x_{i} \in I N\left(f_{2}\right) \backslash I N\left(f_{3}\right)$. Consider an assignment a such that 0 is assigned to $x_{i}$ and 1 is assigned to the other variables. Then, $\hat{f}_{3}(\mathbf{a})=\hat{f}_{4}(\mathbf{a})=1$ and $\hat{f}_{2}(\mathbf{a})=0$ hold.

Case 3: $f_{1}, f_{3}, f_{4}$ are AND functions, and $f_{2}$ is an OR function.

We assume w.l.o.g. that $I N\left(f_{2}\right) \neq I N\left(f_{3}\right)$. We further assume w.l.o.g. that all variables appear positively in $f_{1}, f_{2}$, $f_{3}$, and $f_{4}$.

Let $x_{i} \in I N\left(f_{2}\right) \backslash I N\left(f_{3}\right)$. Consider an assignment a such that 1 is assigned to $x_{i}$ and 0 is assigned to the other variables. Then, $\hat{f}_{3}(\mathbf{a})=\hat{f}_{4}(\mathbf{a})=0$ and $\hat{f}_{2}(\mathbf{a})=1$ hold.

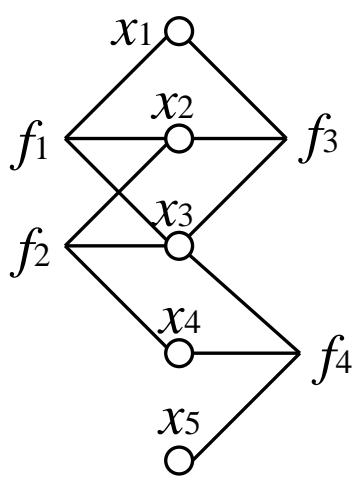

Case 2

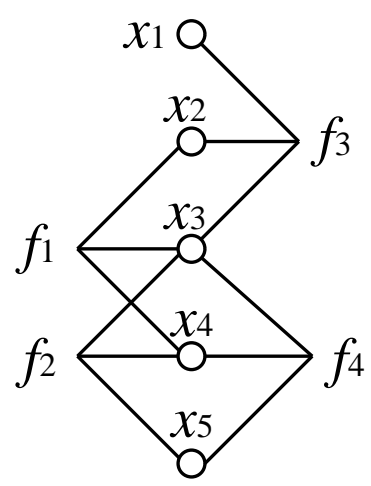

Case 3

Fig. 3. Illustration of Case 2 and Case 3 in the proof of Theorem 1. In Case 2, $\hat{f}_{3}(\mathbf{a})=\hat{f}_{4}(\mathbf{a})=1$ and $\hat{f}_{2}(\mathbf{a})=0$ hold for an assignment $\mathbf{a}=(1,1,1,0,1)$. In Case $3, \hat{f}_{3}(\mathbf{a})=\hat{f}_{4}(\mathbf{a})=0$ and $\hat{f}_{2}(\mathbf{a})=1$ hold for an assignment $\mathbf{a}=(0,0,0,0,1)$.

Case 4: $f_{1}, f_{2}$ are AND functions, and $f_{3}, f_{4}$ is an OR function.

We assume w.l.o.g. that all variables appear positively.

Choose an arbitrary variable $x_{i} \in I N\left(f_{1}\right)$. Consider an assignment a such that 0 is assigned to $x_{i}$ and 1 is assigned to the other variables. Then, $\hat{f}_{3}(\mathbf{a})=\hat{f}_{4}(\mathbf{a})=1$ and $\hat{f}_{1}(\mathbf{a})=$ 0 hold.

Case 5: $f_{1}, f_{3}$ are AND functions, and $f_{2}, f_{4}$ is an OR function.

It is enough to consider the following cases.

Case 5-A: $I N\left(f_{1}\right)=I N\left(f_{3}\right)$ and $I N\left(f_{2}\right)=I N\left(f_{4}\right)$.

Since $\left(f_{1}, f_{2}\right) \neq\left(f_{3}, f_{4}\right)$, we assume w.l.o.g. that there exists $x_{i}$ such that $x_{i}$ appears negatively in $f_{1}$ or $f_{2}$ and all variables in $I N\left(f_{3}\right) \cup I N\left(f_{4}\right)$ appear positively in $f_{3}$ and $f_{4}$. If $x_{i}$ appears negatively in $f_{1}$ (resp., in $f_{2}$ ), consider an assignment a such that 1 (resp., 0 ) is assigned to all variables. Then, $\hat{f}_{3}(\mathbf{a})=\hat{f}_{4}(\mathbf{a})=1$ and $\hat{f}_{1}(\mathbf{a})=0$ (resp., $\hat{f}_{3}(\mathbf{a})=\hat{f}_{4}(\mathbf{a})=0$ and $\hat{f}_{2}(\mathbf{a})=1$ ) hold.

Case 5-B: $I N\left(f_{1}\right) \neq I N\left(f_{3}\right)$ (resp., $I N\left(f_{2}\right) \neq I N\left(f_{4}\right)$ ).

We consider only the case of $I N\left(f_{1}\right) \neq I N\left(f_{3}\right)$, where the other case can be proved in an analogous manner. We assume w.l.o.g. that all variables appear positively in each $f_{i}$.

Let $x_{i} \in I N\left(f_{1}\right) \backslash I N\left(f_{3}\right)$. Consider an assignment a such that 0 is assigned to $x_{i}$ and 1 is assigned to the other variables. Then, $\hat{f}_{3}(\mathbf{a})=\hat{f}_{4}(\mathbf{a})=1$ and $\hat{f}_{1}(\mathbf{a})=0$ hold.

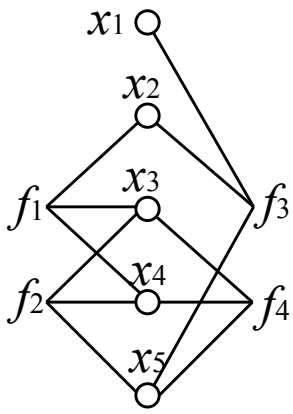

Case 4

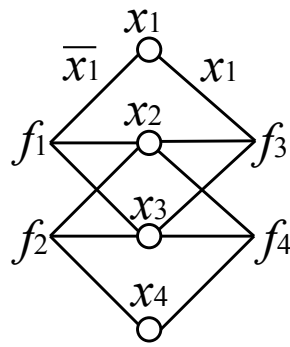

Case 5-A

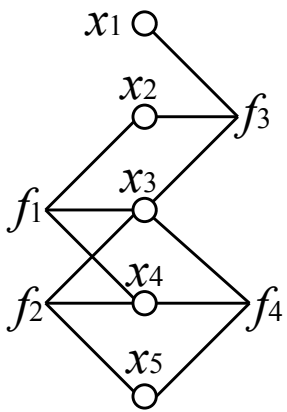

Case 5-B
Fig. 4. Illustration of Case 4 and Case 5 in the proof of Theorem 1. In Case $4, \hat{f}_{3}(\mathbf{a})=\hat{f}_{4}(\mathbf{a})=1$ and $\hat{f}_{1}(\mathbf{a})=0$ hold for an assignment $\mathbf{a}=(1,0,1,1,1)$. In Case 5-A, $\hat{f}_{3}(\mathbf{a})=\hat{f}_{4}(\mathbf{a})=1$ and $\hat{f}_{1}(\mathbf{a})=0$ hold for an assignment $\mathbf{a}=(1,1,1,1)$. In Case 5-B, $\hat{f}_{3}(\mathbf{a})=\hat{f}_{4}(\mathbf{a})=1$ and $\hat{f}_{1}(\mathbf{a})=0$ hold for $\mathbf{a}=(1,1,1,0,1)$.

The theorem cannot be generalized to cases in which a pair of Boolean functions of different degrees is assigned. Consider the case of $f_{1}=x_{1} \wedge x_{2} \wedge x_{3}, f_{2}=x_{2} \wedge x_{3} \wedge x_{4}$, $f_{3}=x_{1} \wedge x_{2}$, and $f_{4}=x_{2} \wedge x_{3} \wedge x_{4}$. Then, any assignment a with $\hat{f}_{3}(\mathbf{a})=\hat{f}_{4}(\mathbf{a})=1$ makes $\hat{f}_{1}(\mathbf{a})=\hat{f}_{2}(\mathbf{a})=1$, and any assignment $\mathbf{a}$ with $\hat{f}_{3}(\mathbf{a})=\hat{f}_{4}(\mathbf{a})=0$ makes $\hat{f}_{1}(\mathbf{a})=$ $\hat{f}_{2}(\mathbf{a})=0$. It is also impossible to generalize the theorem to cases in which a different number of Boolean functions can be assigned. Consider the case of $\left(f_{1}, f_{2}\right)$ and $\left(f_{3}, f_{4}, f_{5}\right)$ 
with $f_{1}=f_{3}=x_{1} \wedge x_{2}, f_{2}=f_{4}=x_{2} \wedge x_{3}, f_{5}=x_{3} \wedge x_{4}$. Since $f_{1}=f_{3}$ and $f_{2}=f_{4}$, samples consistent with $\left(f_{1}, f_{2}\right)$ are always consistent with $\left(f_{3}, f_{4}, f_{5}\right)$.

\section{Full Information MOdel}

For FIM, we consider the class $C_{K}$ of pairs of Boolean functions $\left(f_{1}, f_{2}\right)$ such that $f_{1}=\ell_{s} \vee f_{1}^{\prime}$ and $f_{2}=\ell_{t} \wedge f_{2}^{\prime}$ where $\ell_{r}$ is either $x_{r}$ or $\overline{x_{r}}, x_{s} \neq x_{t}$, and $f_{i}^{\prime}(i=1,2)$ is any Boolean function such that $\left\{x_{s}, x_{t}\right\} \cap I N\left(f_{i}^{\prime}\right)=\{\}$ and $2 \leq\left|I N\left(f_{i}^{\prime}\right)\right| \leq K-1$. This class is called the class of complementary canalyzing pairs (of degree $K$ ). Recall that a Boolean function $f$ is called a canalyzing function if $f$ can be represented as either $f=x_{i} \vee f^{\prime}$ or $f=x_{i} \wedge f^{\prime}$, where it is suggested that most biologically meaningful Boolean functions are canalyzing ones [26]. Then, we have the following theorem.

Theorem 2. For any positive integer $K \geq 3, C_{K}$ is not identifiable from samples under PIM but is identifiable from samples under FIM.

Proof. First, we consider PIM. Let $f_{1}=x_{1} \vee\left(x_{2} \wedge x_{3}\right), f_{2}=$ $x_{4} \wedge\left(x_{2} \vee x_{3}\right), f_{3}=x_{1} \vee x_{2} \vee x_{3}$, and $f_{4}=x_{4} \wedge x_{2} \wedge x_{3}$. Then, it is straight-forward to verify that $\left(f_{3}, f_{4}\right)$ is consistent with any sample generated from $\left(f_{1}, f_{2}\right)$. Therefore, $C_{3}$ (or, $C_{K}$ with $K>3$ ) is not identifiable from samples under PIM.

Next, we consider FIM. Let $f_{1}=x_{s} \vee f_{1}^{\prime}, f_{2}=x_{t} \wedge f_{2}^{\prime}$. The proof can be easily modified for the cases that $x_{s}$ and/or $x_{t}$ appear negatively. In the following $f_{i}=f_{j}$ means that $\hat{f}_{i}(\mathbf{a})=\hat{f}_{j}(\mathbf{a})$ holds for all assignments a. It is to be noted that $f_{i}$ and $f_{j}$ can have different representations.

Let $f_{3}=\ell_{p} \vee f_{3}^{\prime}$ and $f_{4}=\ell_{q} \wedge f_{4}^{\prime}$, where $\ell_{p}$ (resp., $\ell_{q}$ ) is either $x_{p}$ or $\overline{x_{p}}$ (resp., $x_{q}$ or $\overline{x_{q}}$ ). Then, it is enough to show that if $\left(f_{1}, f_{2}\right) \neq\left(f_{3}, f_{4}\right)$, there exists an assignment a such that $\left\{\hat{f}_{1}(\mathbf{a}), \hat{f}_{2}(\mathbf{a})\right\} \neq\left\{\hat{f}_{3}(\mathbf{a}), \hat{f}_{4}(\mathbf{a})\right\}$. We consider the following cases, where symmetric cases are omitted.

\section{Case 1: $x_{s}=x_{p}=\ell_{p}$.}

Suppose that $f_{2} \neq f_{4}$. Since $x_{s}=x_{p}, x_{s} \notin I N\left(f_{2}\right)$ and $x_{s} \notin I N\left(f_{4}\right)$ hold. Then, there exists an assignment a such that $\mathbf{a}[s]=1$ and $\hat{f}_{2}(\mathbf{a}) \neq \hat{f}_{4}(\mathbf{a})$. We assume w.l.o.g. that $\hat{f}_{2}(\mathbf{a})=0$ and $\hat{f}_{4}(\mathbf{a})=1$. Then, we have $\left(\hat{f}_{1}(\mathbf{a}), \hat{f}_{2}(\mathbf{a})\right)=$ $(1,0)$ whereas $\left(\hat{f}_{3}(\mathbf{a}), \hat{f}_{4}(\mathbf{a})\right)=(1,1)$.

Suppose that $f_{2}=f_{4}$. Then, $f_{1} \neq f_{3}$ holds and thus there exists an assignment a such that $\hat{f}_{1}(\mathbf{a}) \neq \hat{f}_{3}(\mathbf{a})$. Since $\hat{f}_{2}(\mathbf{a})=\hat{f}_{4}(\mathbf{a})$ holds, we have $\left\{\hat{f}_{1}(\mathbf{a}), \hat{f}_{2}(\mathbf{a})\right\} \neq$ $\left\{\hat{f}_{3}(\mathbf{a}), \hat{f}_{4}(\mathbf{a})\right\}$.

Case 2: $x_{s}=x_{p}$ and $\ell_{p}=\overline{x_{p}}$.

Consider an assignment $\mathbf{a}$ such that $\mathbf{a}[s]=0, \mathbf{a}[t]=$ 0 , and $\hat{f}_{1}^{\prime}(\mathbf{a})=0$. Then, we have $\left(\hat{f}_{1}(\mathbf{a}), \hat{f}_{2}(\mathbf{a})\right)=(0,0)$ whereas $\left(\hat{f}_{3}(\mathbf{a}), \hat{f}_{4}(\mathbf{a})\right)=(1,0)$ or $\left(\hat{f}_{3}(\mathbf{a}), \hat{f}_{4}(\mathbf{a})\right)=(1,1)$.

Case 3: $x_{s}=x_{q}=\ell_{q}$.

Suppose that there exists an assignment a such that $\mathbf{a}[s]=0, \mathbf{a}[t]=0, \mathbf{a}[p]=1$ and $\hat{f}_{1}^{\prime}(\mathbf{a})=0$. Then, we have $\left(\hat{f}_{1}(\mathbf{a}), \hat{f}_{2}(\mathbf{a})\right)=(0,0)$ whereas $\left(\hat{f}_{3}(\mathbf{a}), \hat{f}_{4}(\mathbf{a})\right)=(1,0)$. Therefore, we can assume w.l.o.g. that $f_{1}^{\prime}=x_{p} \vee f_{1}^{\prime \prime}$ and $f_{1}=x_{s} \vee x_{p} \vee f_{1}^{\prime \prime}$.

Next, suppose that there exists an assignment a such that $\mathbf{a}[s]=1, \mathbf{a}[t]=0, \mathbf{a}[p]=1$ and $\hat{f}_{4}^{\prime}(\mathbf{a})=1$. Then, we have $\left(\hat{f}_{1}(\mathbf{a}), \hat{f}_{2}(\mathbf{a})\right)=(1,0)$ whereas $\left(\hat{f}_{3}(\mathbf{a}), \hat{f}_{4}(\mathbf{a})\right)=(1,1)$. Therefore, we can assume w.l.o.g. that $f_{4}^{\prime}=x_{t} \wedge f_{4}^{\prime \prime}$ and $f_{4}=x_{s} \wedge x_{t} \wedge f_{4}^{\prime \prime}$.

Assuming $f_{1}=x_{s} \vee x_{p} \vee f_{1}^{\prime \prime}$, suppose that there exists an assignment $\mathbf{a}$ such that $\mathbf{a}[s]=0, \mathbf{a}[t]=1, \mathbf{a}[p]=1$ and $\hat{f}_{2}^{\prime}(\mathbf{a})=1$. Then, we have $\left(\hat{f}_{1}(\mathbf{a}), \hat{f}_{2}(\mathbf{a})\right)=(1,1)$ whereas $\left(\hat{f}_{3}(\mathbf{a}), \hat{f}_{4}(\mathbf{a})\right)=(1,0)$. Therefore, we can assume w.l.o.g. that $f_{2}^{\prime}=\overline{x_{p}} \wedge f_{2}^{\prime \prime}$ and $f_{2}=x_{t} \wedge \overline{x_{p}} \wedge f_{2}^{\prime \prime}$.

Here, suppose that there exists an assignment a such that $\mathbf{a}[s]=1, \mathbf{a}[t]=1, \mathbf{a}[p]=1$ and $f_{4}^{\prime \prime}(\mathbf{a})=1$. Then, we have $\left(\hat{f}_{1}(\mathbf{a}), \hat{f}_{2}(\mathbf{a})\right)=(1,0)$ whereas $\left(\hat{f}_{3}(\mathbf{a}), \hat{f}_{4}(\mathbf{a})\right)=(1,1)$.

If $\hat{f}_{4}^{\prime \prime}(\mathbf{a})=0$ holds for all $\mathbf{a}$, we can consider an assignment a such that $\mathbf{a}[s]=1, \mathbf{a}[t]=1$, and $\hat{f}_{2}^{\prime}(\mathbf{a})=1$. Then, we have $\left(\hat{f}_{1}(\mathbf{a}), \hat{f}_{2}(\mathbf{a})\right)=(1,1)$ whereas $\left(\hat{f}_{3}(\mathbf{a}), \hat{f}_{4}(\mathbf{a})\right)=(0,0)$ or $\left(\hat{f}_{3}(\mathbf{a}), \hat{f}_{4}(\mathbf{a})\right)=(1,0)$.

Case 4: $x_{s}=x_{q}$ and $\ell_{q}=\overline{x_{q}}$.

Consider an assignment $\mathbf{a}$ such that $\mathbf{a}[s]=1, \mathbf{a}[t]=$ 1 , and $\hat{f}_{2}^{\prime}(\mathbf{a})=1$. Then, we have $\left(\hat{f}_{1}(\mathbf{a}), \hat{f}_{2}(\mathbf{a})\right)=(1,1)$ whereas $\left(\hat{f}_{3}(\mathbf{a}), \hat{f}_{4}(\mathbf{a})\right)=(0,0)$ or $\left(\hat{f}_{3}(\mathbf{a}), \hat{f}_{4}(\mathbf{a})\right)=(1,0)$.

Case 5: $x_{s} \neq x_{p}, x_{s} \neq x_{q}, x_{t} \neq x_{p}, x_{t} \neq x_{q}$.

We consider the case of $x_{s}=\ell_{s}, x_{t}=\ell_{t}, x_{p}=\ell_{p}$, and $x_{q}=\ell_{q}$, where the other cases can be proven in a similar way.

If $x_{p} \notin I N\left(f_{1}^{\prime}\right)$, we can consider an assignment a such that $\mathbf{a}[s]=0, \mathbf{a}[t]=0, \mathbf{a}[p]=1$, and $\hat{f}_{1}^{\prime}(\mathbf{a})=0$. Then, we have $\left(\hat{f}_{1}(\mathbf{a}), \hat{f}_{2}(\mathbf{a})\right)=(0,0)$ whereas $\left(\hat{f}_{3}(\mathbf{a}), \hat{f}_{4}(\mathbf{a})\right)=(1,0)$ or $\left(\hat{f}_{3}(\mathbf{a}), \hat{f}_{4}(\mathbf{a})\right)=(1,1)$.

In the following, we assume w.l.o.g. that $x_{p} \in I N\left(f_{1}^{\prime}\right)$. Suppose that there exists an assignment a such that $\mathbf{a}[s]=0$, $\mathbf{a}[t]=0, \mathbf{a}[p]=1$, and $\hat{f}_{1}^{\prime}(\mathbf{a})=0$. Then, we have $\left(\hat{f}_{1}(\mathbf{a}), \hat{f}_{2}(\mathbf{a})\right)=(0,0)$ whereas $\left(\hat{f}_{3}(\mathbf{a}), \hat{f}_{4}(\mathbf{a})\right)=(1,0)$ or $\left(\hat{f}_{3}(\mathbf{a}), \hat{f}_{4}(\mathbf{a})\right)=(1,1)$. Therefore, we can assume that $f_{1}$ has the form of $f_{1}=x_{s} \vee x_{p} \vee f_{1}^{\prime \prime}$.

Suppose that $x_{s} \notin I N\left(f_{3}^{\prime}\right)$. We can consider an assignment a such that $\mathbf{a}[s]=1, \mathbf{a}[p]=0, \mathbf{a}[q]=0$, and $\hat{f}_{3}^{\prime}(\mathbf{a})=0$. Then, we have $\left(\hat{f}_{1}(\mathbf{a}), \hat{f}_{2}(\mathbf{a})\right)=(1,0)$ or $\left(\hat{f}_{1}(\mathbf{a}), \hat{f}_{2}(\mathbf{a})\right)=(1,1)$ whereas $\left(\hat{f}_{3}(\mathbf{a}), \hat{f}_{4}(\mathbf{a})\right)=(0,0)$. Therefore, we assume w.l.o.g. that $x_{s} \in I N\left(f_{3}^{\prime}\right)$ holds. Then, $f_{3}$ has the form of $f_{3}=x_{s} \vee x_{p} \vee f_{3}^{\prime \prime}$ as in the case of $f_{1}$.

If $f_{1}^{\prime \prime}=f_{3}^{\prime \prime}$ (i.e., $f_{1}=f_{3}$ ), we can prove the theorem as in Case 1. Therefore, we assume w.l.o.g. that $f_{1}^{\prime \prime} \neq f_{3}^{\prime \prime}$. Then, there exists an assignment a such that $\mathbf{a}[s]=\mathbf{a}[p]=0, \hat{f_{1}^{\prime \prime}}(\mathbf{a})=0$, and $\hat{f}_{3}^{\prime \prime}(\mathbf{a})=1$. If $\mathbf{a}[t]=0$, we have $\left(\hat{f}_{1}(\mathbf{a}), \hat{f}_{2}(\mathbf{a})\right)=(0,0)$ whereas $\left(\hat{f}_{3}(\mathbf{a}), \hat{f}_{4}(\mathbf{a})\right)=$ $(1,0)$ or $\left(\hat{f}_{3}(\mathbf{a}), \hat{f}_{4}(\mathbf{a})\right)=(1,1)$. If there exists no such assignment, $f_{3}^{\prime \prime}$ has the form of $f_{3}^{\prime \prime}=x_{t} \wedge f_{3}^{\prime \prime \prime}$ and thus $f_{3}=\left(x_{s} \vee x_{p}\right) \vee\left(x_{t} \wedge f_{3}^{\prime \prime \prime}\right)$. Similarly, we can assume w.l.o.g. that $f_{1}=\left(x_{s} \vee x_{p}\right) \vee\left(x_{q} \wedge f_{1}^{\prime \prime \prime}\right), f_{2}=\left(x_{t} \wedge x_{q}\right) \wedge\left(x_{p} \vee f_{2}^{\prime \prime \prime}\right)$, and $f_{4}=\left(x_{t} \wedge x_{q}\right) \wedge\left(x_{s} \vee f_{4}^{\prime \prime \prime}\right)$. Then, there exists an assignment a such that either $f_{1}^{\prime \prime \prime}(\mathbf{a})=1, \hat{f_{2}^{\prime \prime \prime}}(\mathbf{a})=0$, $f_{3}^{\prime \prime \prime}(\mathbf{a})=1$, or $f_{4}^{\prime \prime \prime}(\mathbf{a})=0$ holds, otherwise $f_{1}=f_{3}$ and $f_{2}=f_{4}$ would hold. We can assume w.l.o.g. that $\hat{f}_{1}^{\prime \prime \prime}(\mathbf{a})=1$ holds. Consider the assignment $\mathbf{a}^{\prime}=\mathbf{a}_{-s,-p,-t,+q}$. Then, we have $\left(\hat{f}_{1}\left(\mathbf{a}^{\prime}\right), \hat{f}_{2}\left(\mathbf{a}^{\prime}\right)\right)=(1,0)$ whereas $\left(\hat{f}_{3}\left(\mathbf{a}^{\prime}\right), \hat{f}_{4}\left(\mathbf{a}^{\prime}\right)\right)=$ $(0,0)$.

We provide an example showing that Theorem 2 cannot be generalized to the class of PBNs of nested canalyzing 
TABLE 1

Difficult case for nested canalyzing functions under FIM.

\begin{tabular}{|c|cccccccccccccccc|}
\hline$x_{1}$ & 0 & 0 & 0 & 0 & 0 & 0 & 0 & 0 & 1 & 1 & 1 & 1 & 1 & 1 & 1 & 1 \\
\hline$x_{2}$ & 0 & 0 & 0 & 0 & 1 & 1 & 1 & 1 & 0 & 0 & 0 & 0 & 1 & 1 & 1 & 1 \\
\hline$x_{3}$ & 0 & 0 & 1 & 1 & 0 & 0 & 1 & 1 & 0 & 0 & 1 & 1 & 0 & 0 & 1 & 1 \\
\hline$x_{4}$ & 0 & 1 & 0 & 1 & 0 & 1 & 0 & 1 & 0 & 1 & 0 & 1 & 0 & 1 & 0 & 1 \\
\hline$f_{1}$ & 0 & 0 & 0 & 0 & 0 & 0 & 1 & 1 & 0 & 0 & 0 & 0 & 1 & 1 & 1 & 1 \\
\hline$f_{2}$ & 0 & 0 & 1 & 1 & 0 & 1 & 1 & 1 & 0 & 0 & 1 & 1 & 0 & 1 & 1 & 1 \\
\hline Output & 0 & 0 & 0,1 & 0,1 & 0 & 0,1 & 1 & 1 & 0 & 0 & 0,1 & 0,1 & 0,1 & 1 & 1 & 1 \\
\hline$f_{3}$ & 0 & 0 & 1 & 1 & 0 & 0 & 1 & 1 & 0 & 0 & 1 & 1 & 1 & 1 & 1 & 1 \\
\hline$f_{4}$ & 0 & 0 & 0 & 0 & 0 & 1 & 1 & 1 & 0 & 0 & 0 & 0 & 0 & 1 & 1 & 1 \\
\hline
\end{tabular}

function pairs consisting of only positive literals. Let $K=$ $3, n=4$, consider two pairs of Boolean functions $\left(f_{1}, f_{2}\right)=$ $\left(x_{2} \wedge\left(x_{1} \vee x_{3}\right), x_{3} \vee\left(x_{2} \wedge x_{4}\right)\right)$ and $\left(f_{3}, f_{4}\right)=\left(x_{3} \vee\left(x_{1} \wedge\right.\right.$ $\left.\left.x_{2}\right), x_{2} \wedge\left(x_{4} \vee x_{3}\right)\right)$. Then, $\left\{\hat{f}_{1}(\mathbf{a}), \hat{f}_{2}(\mathbf{a})\right\}=\left\{\hat{f}_{3}(\mathbf{a}), \hat{f}_{4}(\mathbf{a})\right\}$ holds for all $\mathbf{a} \in\{0,1\}^{4}$ as shown in Table 1, where " 0,1 " means that we have both outputs.

\section{SAMPle Complexity}

In the above, we have shown that the classes of admissible AND/OR pairs and complementary canalyzing pairs can be identified under PIM and FIM, respectively Here, we analyze how many samples are required in order to uniquely identify a PBN under the following assumptions:

- a sample is a pair $(\mathbf{a}, \mathbf{b})$ of $0-1$ assignment to $n$ variables and 0-1 output values of $n$ nodes,

- each 0-1 assignment is generated uniformly at random,

- the output value of each node is determined according to a pair of Boolean functions assigned to the node in an underlying PBN of the target class, where selection of a function from the pair is independently done for each assignment with probability $1 / 2$,

- each sample is generated independently.

Note that $\mathbf{a}$ and $\mathbf{b}$ in a sample $(\mathbf{a}, \mathbf{b})$ correspond to global states of a PBN at time steps $t$ and $t+1$, respectively, although samples are independent from each other. Using Theorem 1, Theorem 2, and a similar argument as in [9], we have the following theorem.

Theorem 3. For the class of PBNs consisting of admissible AND/OR pairs of degree $K$ (resp., complementary canalyzing pairs of degree $K)$, if $O\left(2^{4 K+1}(4 K+\alpha) \log n\right)$ samples are given uniformly at random, the correct PBN can be uniquely identified with probability at least $1-\frac{1}{n^{\alpha}}$ under PIM (resp., FIM).

Proof. We prove for the case of PIM, where the case of FIM can be proved in an analogous manner.

Suppose that $\left(f_{1}, f_{2}\right)$ is the underlying function pair for $k$-th node in a PBN. It is seen from the proof of Theorem 1 that if all possible assignments on $I=I N\left(f_{1}\right) \cup I N\left(f_{2}\right) \cup$ $I N\left(f_{3}\right) \cup I N\left(f_{4}\right)$ and their possible output values by $f_{1}$ and $f_{2}$ are given, inconsistency of $\left(f_{3}, f_{4}\right)\left(\neq\left(f_{1}, f_{2}\right)\right)$ can be detected. Since $|I| \leq 4 K$, it is also seen that if all possible assignments on all possible combination of $4 K$ variables and their possible output values appear in samples (Condition C1), we can uniquely identify $\left(f_{1}, f_{2}\right)$.
The probability that $\mathbf{a}[i]=1$ for $i=1, \ldots, 4 K$ and $\mathbf{b}[k]=\hat{f}_{1}(\mathbf{a})$ do not hold in a given sample $(\mathbf{a}, \mathbf{b})$ is at most $1-\frac{1}{2^{4 K+1}}$, and thus the same condition does not hold in any $m$ samples is at most $\left(1-\frac{1}{2^{4 K+1}}\right)^{m}$. Since the number of combination of $4 K$ variables is less than $n^{4 K}$, the probability that Condition $\mathrm{C} 1$ does not hold is bounded above by $2^{4 K+1} \cdot n^{4 K} \cdot\left(1-\frac{1}{2^{4 K+1}}\right)^{m}$. Since there exist $n$ nodes, the probability that Condition $\mathrm{C} 1$ does not hold for one or more nodes is bounded above by

$$
p_{K, n, m}=2^{4 K+1} \cdot n^{4 K+1} \cdot\left(1-\frac{1}{2^{4 K+1}}\right)^{m} .
$$

It is not difficult to see that $p_{K, n, m} \leq p$ holds if

$$
m>\ln 2 \cdot 2^{4 K+1}\left((4 K+1)(1+\log n)+\log \frac{1}{p}\right) .
$$

Letting $p=\frac{1}{n^{\alpha}}$, the theorem holds.

\section{Computational Experiments}

In order to verify the result of Theorem 3, we performed computational experiments. Since it is impossible under FIM to know whether a given sample set is enough, we only examined the case of PIM. In the experiments, we examined the cases of $K=1$ and $K=2$, where both types of PBNs consisting of AND functions (AND PBNs) and AND/OR functions (AND/OR PBNs) were tested for $K=2$. For each $n$ and $K$, we randomly generated 100 PBNs. Then, for each PBN, we generated samples by assigning 0 or 1 to each node with probability $1 / 2$ and updating the PBN synchronously to obtain output values. Finally, we computed the average number of samples required to uniquely identify each PBN. For that purpose, we examined samples one-by-one until the number of consistent PBNs became 1 , where we maintained consistent Boolean function pairs independently for each node. The programs for generation and identification of PBNs were implemented using Python language, whereas simulation of PBNs was performed using 'BoolNet' [27]. All experiments were performed on a PC cluster with Intel(R) Xeon(R) CPU E5-2690 2.90GHz and 35.87 GB memory.

The results are shown in Fig. 5. For the case of $K=1$ (Fig. $5(a)), n$ is varied from 5 to 100 . It seems that the number of samples is proportional to $\log n$. Even for the case of $n=100$, approximately only 100 samples were required to identify PBNs. Although the computation time 


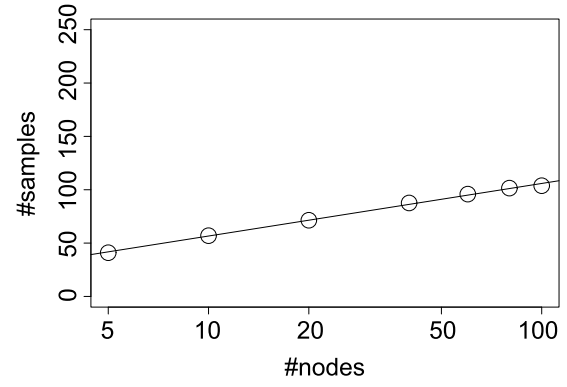

(a) $K=1$

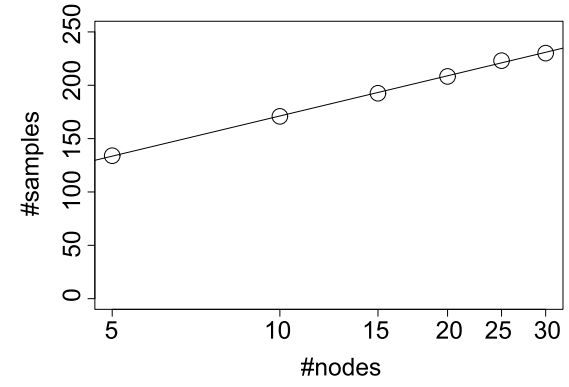

(b) $K=2$ (AND PBNs)

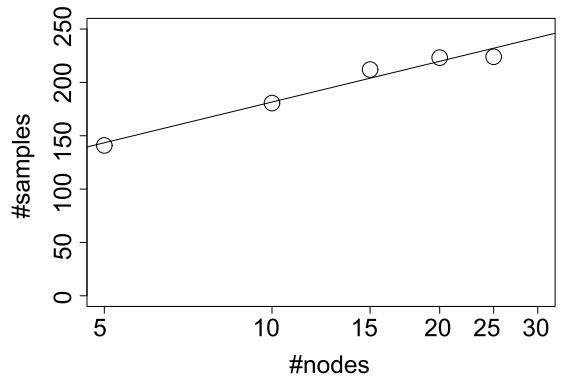

(c) $K=2(\mathrm{AND} / \mathrm{OR} \mathrm{PBNs})$

Fig. 5. Results of computational experiments. (a), (b) and (c) show the number of samples required to identify PBNs of the target class under PIM for the cases of $K=1, K=2$ (AND PBNs) and $K=2$ (AND/OR PBNs), respectively. Regression lines are indicated within each panel, where $x$-axis is log-scaled.

for identification increased rapidly as $n$ grew, it took around only 30 seconds per identification of a PBN.

Similarly, for the cases of $K=2$ (AND PBNs) (Fig. 5(b)) and $K=2$ (AND/OR PBNs) (Fig. 5(c)), the number of required samples also looks to be proportional to $\log n$. However, it took long computation time: approximately 12 hours and 36 hours per identification of a PBN for the largest cases of AND PBNs $(n=30)$ and AND/OR PBNs $(n=25)$, respectively. For this reason, we could not perform computational experiments for much larger $n$ and $K$. It is also to be noted that the numbers of required samples for AND PBNs and AND/OR PBNs were not so different. This is reasonable because the set of all possible Boolean functions per node for an AND/OR PBN is at most three times larger than that for an AND PBNs.

\section{CONCLUSION}

We have studied the number of samples in order to exactly identify the structure of a PBN from samples, with focusing on the case where two Boolean functions are assigned to each node. We considered two models: partial information model (PIM) and full information model (FIM). We showed that it is possible to identify the structure of a PBN from a small number $(O(\log n))$ of samples under both models for reasonably wide classes of PBNs whereas there exist classes of PBNs whose structure cannot be identified from samples. In addition, the positive result on PIM was verified via computational experiments. These results are interesting because they show that the structure of a probabilistic system can be exactly identified to some extent. It is to be noted that these results are independent of inference algorithms. It was also shown that there is a large gap of identifiable classes between PIM and FIM. The merit of PIM is that we can know when the structure is uniquely identified. However, FIM allows us to identify the structure for a much wider class of PBNs. Therefore, if an enough number of samples are available, it is better to use FIM.

Although we obtained fundamental results on the sample complexity for identification of a PBN, there are many things to be explored. One important thing is to study the cases in which more than two Boolean functions are assigned per node. Another important thing is to make use of the probabilities assigned to Boolean functions. In order to show negative results, we assumed that the probability assigned to each function is $1 / 2$. However, if different probabilities are assigned, we may identify the structure of a PBN for wider classes of PBNs. In addition to the sample complexity issue, it is also important to develop efficient identification algorithms because we employed a naive enumeration-based algorithm in computational experiments and thus could not handle large-scale PBNs even for $K=2$. Further studies might lead to much deeper understanding of complex biological networks.

\section{ACKNOWLEDGMENTS}

This work was partially supported by Grant-in-Aid \#26540125 from JSPS, Japan. We thank Avraham Melkman for helpful discussions.

\section{REFERENCES}

[1] S. A. Kauffman, "Metabolic stability and epigenesis in randomly constructed genetic nets," J. Theor. Biol., vol. 22, pp. 437-467, 1969.

[2] S. A. Kauffman, The Origins of Order: Self-Organization and Selection in Evolution. New York:Oxford University Press, 1993.

[3] B. Samuelsson and C. Troein, "Superpolynomial growth in the number of attractors in Kauffman networks," Phys. Rev. Lett., vol. 90, 098701, 2003.

[4] B. Drossel, T. Mihaljev, and F. Greil, "Number and length of attractors in a critical Kauffman model with connectivity one," Phys. Rev. Lett., vol. 94, 088701, 2005.

[5] P. Krawitz and I. Shmulevich, "Basin entropy in Boolean network ensembles," Phys. Rev. Lett., vol. 98, 158701, 2007.

[6] C. Seshadhri, Y. Vorobeychik, J. R. Mayo, R. C. Armstrong, and J. R. Ruthruff, "Influence and dynamic behavior in random Boolean networks," Phys. Rev. Lett., vol. 107, 108701, 2011.

[7] S. Squires, E. Ott, and M. Girvan, "Dynamical instability in Boolean networks as a percolation problem," Phys. Rev. Lett., vol. 109, 085701, 2012.

[8] S. Liang, S. Fuhrman, and R. Somogyi, "REVEAL, a general reverse engineering algorithm for inference of genetic network architectures," in Proceedings of Pacific Symposium on Biocomputing 1998, pp. 18-29, Singapore:World Scientific, 1998.

[9] T. Akutsu, S. Miyano, and S. Kuhara, "Identification of genetic networks from a small number of gene expression patterns under the Boolean network model," in Proceedings of Pacific Symposium on Biocomputing 1999, Singapore:World Scientific, pp. 17-28. 1999.

[10] R. Laubenbacher and B. Stigler, "A computational algebra approach to the reverse engineering of gene regulatory networks," $J$. Theor. Biol., vol. 229, pp. 523-537, 2004.

[11] T. J. Perkins and M. T. Hallett, "A trade-off between sample complexity and computational complexity in learning Boolean networks from time-series data," IEEE/ACM Trans. Comput. Biol. Bioinform., vol. 7, pp. 118-128, 2010. 
[12] P. Vera-Licona1, A. S. Jarrah, L. D. Garcia-Puente, J. McGee, and $\mathrm{R}$, Laubenbacher, "An algebra-based method for inferring gene regulatory networks." BMC Syst. Biol., vol. 8, 37, 2014.

[13] E. N. Miranda and N. Parga, "Noise effects in the Kauffman model," Europhys. Lett., vol. 10, pp. 293-298, 1989.

[14] T. Akutsu, S. Miyano, and S. Kuhara, "Inferring qualitative relations in genetic networks and metabolic pathways," Bioinformatics, vol. 16, pp. 727-734, 2000.

[15] T. P. Peixoto, "Redundancy and error resilience in Boolean networks," Phys. Rev. Lett., vol. 104, 048701, 2010.

[16] I. Shmulevich, E. R. Dougherty, S. Kim, and W. Zhang, "Probabilistic Boolean networks: a rule-based uncertainty model for gene regulatory networks," Bioinformatics, vol. 18, pp. 261-274, 2001.

[17] I. Shmulevich and E. R. Dougherty, Probabilistic Boolean Networks: The Modeling and Control of Gene Regulatory Networks. Philadelphia:SIAM, 2010.

[18] A. Datta, A. Choudhary, M. L. Bittner, and E. R. Dougherty, “External control in Markovian genetic regulatory networks," Machine Learning, vol. 52, pp. 169-191, 2003.

[19] W-K. Ching, S. Zhang, M. Ng, and T. Akutsu, "An approximation method for solving the steady-state probability distribution of probabilistic Boolean networks," Bioinformatics, vol. 23, pp. 15111518, 2007.

[20] B. Faryabi, G. Vahedi, A. Datta, J. F. Chamberland, and E. R. Dougherty, "Curr. Genomics, vol. 10, pp. 463-477, 2009.

[21] K. Kobayashi and K. Hiraishi, "An integer programming approach to optimal control problems in context-sensitive probabilistic Boolean networks," Automatica, vol. 47, pp. 1260-1264, 2011.

[22] P. Trairatphisan, A. Mizera, J. Pang, A. A. Tantar, J. Schneider, and T. Sauter, "Recent development and biomedical applications of probabilistic Boolean networks," Cell Commun. Signal., vol. 11, 46, 2013.

[23] S. Marshall, L. Yu, Y. Xiao, and E. R. Dougherty, "Inference of a probabilistic Boolean network from a single observed temporal sequence," EURASUP J. Bioinform. Syst. Biol., vol. 2007, 32454, 2007.

[24] N. Friedman and Z. Yakhini, "On the sample complexity of learning Bayesian networks," in Proceedings of 20th Conf. Uncertainty in Artificial Intelligence pp. 274-282, CA:Morgan Kaufmann, 1996.

[25] S. Dasgupta, "The sample complexity of learning fixed-structure Bayesian networks," Mach. Learn., vol. 29, pp. 165-180, 1997.

[26] S. E. Harris, B. K. Sawhill, A. Wuensche, and S. Kauffman, "A model of transcriptional regulatory networks based on biases in the observed regulation rules," Complexity, vol. 7, pp. 23-40, 2002.

[27] C. Müssel, M. Hopfensitz, and H. Kestler, "Boolnet-an R package for generation, reconstruction and analysis of boolean networks," Bioinformatics, vol. 26, pp. 1378-1380, 2010.

Xiaoqing Cheng was born in Hangzhou, China in 1990. She received the B.E degree from Department of Mathematics, University of Science and Technology of China, Hefei, China in 2012 and has been a Ph.D student in Department of Mathematics, The University of Hong Kong from 2012.09. Her main areas of research interest are bioinformatics and data mining.

Tomoya Mori received B.E. and M.E. degrees in informatics from Doshisha University and Kyoto University in 2010 and 2012, respectively. He is currently a doctor course student at Kyoto University, Japan. His research interests are in bioinformatics and discrete algorithms.

Yushan Qiu was born in Guangdong, China, in 1988. She received the B.E. degree in School of Mathematics from the South China Normal University, Guangdong, China, in 2007. She has been a PhD student in the Department of Mathematics, The University of Hong Kong since 2011. Her research interests include Bioinformatics and machine learning.
Wai-Ki Ching Wai-Ki Ching is a Professor and the head of department in the Department of Mathematics, University of Hong Kong. He obtained his B. Sci. (Hons) and M. Phil. degrees from the University of Hong Kong. He then received his Ph.D. degree from the Chinese University of Hong Kong. His research interests are stochastic modelling and matrix computations. In particular, the applications of mathematical models and numerical algorithms in solving problems related to Markov chains, bioinformatics, systems biology and management science.

Tatsuya Akutsu received the B.E. and M.E. degrees in aeronautics and the D.E. degree in information engineering from the University of Tokyo, 1984,1986 , and 1989, respectively. Since 2001 , he has been a professor in the Bioinformatics Center, Institute for Chemical Research, Kyoto University. His research interests include bioinformatics and discrete algorithms. His research interests include bioinformatics and discrete algorithms. He is a member of the IEEE. 